\title{
ANALYSIS OF MUSCLE ACTIVITY DURING THE BENCH PRESS EXERCISE PERFORMED WITH THE PRE-ACTIVATION METHOD ON STABLE AND UNSTABLE SURFACES
}

\author{
André Luiz Torres Pirauá1,3, Natália Barros Beltrão, ${ }^{2,3}$, Camila Ximenes Santos ${ }^{3}$, \\ Ana Carolina Rodarti Pitangui ${ }^{4}$, and Rodrigo Cappato de Araújo ${ }^{3,4}$ \\ ${ }^{1}$ Department of Physical Activity - Asces College, Caruaru - PE, Brazil \\ ${ }^{2}$ Department of Physical Activity - Federal Rural University of Pernambuco, Recife - PE, Brazil \\ ${ }^{3}$ Physical Education Associated Post - Graduation Program UPE/UFPB, Recife - PE, Brazil \\ ${ }^{4}$ Department of Physiotherapy, University of Pernambuco, Petrolina - PE, Brazil
}

Original scientific paper

UDC: $796.015: 612$

\begin{abstract}
:
This study compared the EMG activity of the upper limb and shoulder girdle muscles in a resistance training session involving the pre-activation method in stable and unstable conditions. Fourteen healthy male volunteers (age 22.5 \pm 2.4 years; body height $173.6 \pm 7.10 \mathrm{~cm}$; body mass $76.03 \pm 9.02 \mathrm{~kg}$ ) performed under three exercise conditions in a randomized order: a) barbell bench press, b) stable dumbbell fly and barbell bench press, and c) unstable dumbbell fly and barbell bench press. Subjects performed 10 repetitions of stable or unstable dumbbell fly at $30 \%$ of $1 \mathrm{RM}$, and barbell bench press was executed at $60 \%$ of $1 \mathrm{RM}$ until concentric failure. The 1RM test was performed for both exercises and, in case of dumbbell fly, on both surfaces. EMG-signals from the m. pectoralis maior (PM), deltoid anterior (DA), triceps brachii (TB) and serratus anterior (SA) were acquired. Two-way ANOVA for repeated measures indicated that the pre-activation method performed on both surfaces raised the EMG activity for all muscles: $\mathrm{PM}(\mathrm{p} \leq 0.01), \mathrm{DA}(\mathrm{p} \leq 0.02), \mathrm{TB}(\mathrm{p} \leq 0.02)$ and SA ( $p \leq 0.03)$, and no differences were observed between the stable and unstable conditions $(p \geq 0.228)$. These results indicate that the execution of a previous exercise increases EMG activity. However, as there was no exclusive increase for primary agonist muscles, this increase cannot be attributed to the pre-activation method but rather to the completion of two consecutive exercises.
\end{abstract}

Key words: resistance training, electromyography, muscle strength, instability, shoulder

\section{Introduction}

The enhanced strength adaptations of muscles, induced by resistance training (RT), are associated with the combination of neural factors and muscle hypertrophy (Ribeiro, et al., 2013). However, these adjustments tend to occur to a lesser extent as the subject becomes more trained, and, if an appropriate technical training progression has not been applied, the subject can enter into a training plateau (ACSM, 2009). In this sense, the manipulation of training variables (intensity, volume, frequency, rest period length, or exercise selection and order) shall be adopted as a RT alternative in order to get positive responses in muscle performance (Ribeiro, et al., 2013).

The pre-exhaustion method suggests combining two exercises for the same muscle group in a way that a single joint exercise precedes a multi-joint exercise (Brennecke, et al., 2009; Júnior, et al., 2010). It is believed that the realization of this method favors a greater recruitment of the prime movers in the multi-joint exercise (Fleck \& Kraemer, 2004). Most of the studies that tested the effectiveness of the pre-exhaustion method did not find differences (Brennecke, et al., 2009; Gentil, Oliveira, Júnior, do Carmo, \& Bottaro, 2007) or decreases in the level of electromyographic (EMG) activity of the prime movers (Augustsson, et al., 2003). It is important to note that all these studies used high loads in both single and multi-joint exercises.

On the other hand, Júnior et al. (2010) used submaximal intensity was used in the single-joint exercise (60 and 30\% 1RM), and identified a higher EMG amplitude for the agonist muscle. Therefore, the authors denominated the method as pre-activation, considering that they did not use maximum 
repetitions during the single-joint exercise. Thus, it is believed that increases in agonist muscle activation occur only when submaximal intensities are used in a single-joint exercise.

Furthermore, the inclusion of exercises with instability has been seen as an alternative to RT stimuli variation (Andrade, Araújo, Tucci, Martins, \& Oliveira, 2011; Behm \& Colado, 2012; Tucci, et al., 2011). The use of unstable surfaces in RT is based on the idea that instability promotes a greater neuromuscular challenge due to the need of stabilization during exercise execution (Behm \& Colado, 2012; de Araújo, et al., 2009; Melo, Pirauá, Beltrão, Pitangui, \& Araújo, 2014; Pirauá, et al., 2014). Whereas the pre-activation method and the use of unstable surfaces are both used to increase the neuromuscular demand, it is possible to speculate that the pre-activation method combined with an unstable surface provides a greater muscle activity when performing a multi-joint exercise. Therefore, the aim of this study was to compare the EMG activity of the upper limb muscles and shoulder girdle in a RT session involving the pre-activation method under stable and unstable conditions.

\section{Methods}

\section{Subjects}

Fourteen healthy young male volunteers took part in the study (age $22.5 \pm 2.4$ years; body height $173.6 \pm 7.10 \mathrm{~cm}$; body mass $76.03 \pm 9.02 \mathrm{~kg}$ ). All subjects were: a) experienced in resistance training for at least six months and b) fit to do exercises according to the Physical Activity Readiness Questionnaire. Volunteers were excluded if they had practiced any type of exercise $48 \mathrm{~h}$ before an experimental or testing session and/or had not completed any phase of the study. The study had the institutional ethical board approval and was conducted in accordance with the recognized national and international ethical standards. Subjects were informed of the benefits and risks of the investigation prior to signing a written informed consent form.

\section{Procedures}

EMG-signals from the pectoralis maior (PM), deltoid anterior (DA), triceps brachii (TB) and serratus anterior (SA) muscles were acquired. Myoelectric activity was registered using surface differential and simple electrodes with a gain of 20 times, consisting of two parallel rectangular pure silver bars $(10 \times 2 \times 1 \mathrm{~mm}$, with a $20 \mathrm{~mm}$ interelectrode distance, input impedance of $10 \mathrm{GX}$ and common mode rejection ratio of $130 \mathrm{~dB}$ ) (Datahominis Tecnologia Ltda ${ }^{\circledR}$, Uberlândia - Brazil). Skin was shaved at the electrode sites, gently abraded and cleaned with alcohol to reduce skin impedance. The surface electrodes were positioned with tape longitudinally over the muscle belly, approximately in the direction of the muscle fibers, according to the recommendations of SENIAM (Hermens, Freriks, Disselhorst-Klug, \& Rau, 2000).

A Myosystem Br-1 version 3.5 (Datahominis Tecnologia Ltda ${ }^{\circledR}$, Uberlândia - Brazil) was used for the initial EMG signal processing. Thereafter, data were sampled by a 16-bit A/D converter board (input voltage of $-10 \mathrm{~V}$ to $+10 \mathrm{~V}$ ) with a $2000 \mathrm{~Hz}$ frequency and band-pass filtered at $10 \mathrm{~Hz}-5 \mathrm{kHz}$. Raw EMG data were digitally filtered at a frequency bandwidth between 15 and $500 \mathrm{~Hz}$, and the root mean square (RMS) value was calculated in sliding windows of $100 \mathrm{~ms}$. The first and last repetition of each exercise was excluded. Thus, EMG activity data were the average of the intermediate repetition values.

Exercise kinematic data were assessed using a digital camera (SONY, DCR-SX21 model) with an acquisition capacity of $60 \mathrm{~Hz}$ and processed in free software (VirtualDub 1.9.11 - VirtualDub. org). The camera was fixed to a tripod and placed at a distance of two meters from the subject in the subject's cranial-caudal direction in the transverse plane. Kinematic analysis was used to identify exercise phases (concentric and eccentric). A synchronization system (DataHominis Technology Ltda ${ }^{\circledR}$, Uberlandia - Brazil) was used for synchronizing kinematic and electromyography systems. This system synchronously triggers a pulse to the EMG system and a light signal to the recording system. Thus, it was possible to establish cutoff points for each complete movement cycle for both exercises.

Each subject attended two experimental sessions, separated by 2-7 days, in a counterbalanced design. On day 1, anthropometric measures were collected, and the 1RM test for the barbell bench press, stable dumbbell fly (on a bench) and unstable dumbbell fly (on a Swiss ball) was performed following the Brown and Weir (2001) protocol. The 1RM test started with a specific warm-up, which included one set of five repetitions at $50 \%$ of maximal load (estimated by the subject) and one set of three repetitions at $80 \%$ of maximal load (estimated by the subject) with a three-minute interval between them. The initial load for each 1RM test was estimated by the volunteer. After the first attempt, the loads were adjusted with increments ranging from 2 to $10 \mathrm{~kg}$. For correct execution, subjects were in a supine position (over the bench or with the head and shoulders resting completely on the ball) with the feet parallel and placed on the ground. Subjects had three to five trials with a five-minute interval to establish maximal load.

On the second visit, the experimental session was conducted. Initially, electrodes were positioned on the right side of the subject. Maximal voluntary isometric contraction (MVIC) tests for each assessed muscle was executed for normalizing the EMG signals. The MVIC tests were performed on 
a muscle function with manual resistance (Kendall, Kendall, \& Wadsworth, 1973) according to the priority muscle action. For each test, subjects performed three repetitions of six seconds with a 90 -second interval between them. The muscle testing sequence was randomized.

Ten minutes after the MVIC tests, the subjects started the exercises. Each volunteer executed three exercises: a) bench press (traditional method), b) stable dumbbell fly + bench press (pre-activation method), and c) unstable dumbbell fly + bench press (pre-activation method). The bench press was performed on a horizontal bench using a barbell, and the subject had to be in a supine position with the feet parallel and placed on the floor. During the exercise, the subject had to touch the chest with a thin mattress (placed on the barbell) to guarantee that the elbow flexion was standardized at $90^{\circ}$ (Figure 1). For the barbell dumbbell fly execution, subjects were in a supine position on the bench (stable) or over the ball (unstable) with the feet parallel and placed on the floor and the head placed on the surface (Figures 2 and 3). The order in which subjects executed the protocols was randomized, and a 10-minute interval was standardized.

For all three protocols, a metronome controlled velocity of execution with $3 \mathrm{~s}$ for each repetition (1 $\mathrm{s}$ concentric phase / $2 \mathrm{~s}$ eccentric phase). Intensity was set at $30 \%$ of $1 \mathrm{RM}$ for the stable and unstable dumbbell fly and $60 \%$ of 1RM for the bench press. Regarding the number of repetitions for the stable and unstable dumbbell fly, the subjects performed 10 repetitions of each exercise, and bench press repetitions were executed until concentric failure.



Figure 1. Side view of the movement's initial phase (A) and final phase (B) of the barbell horizontal bench press.

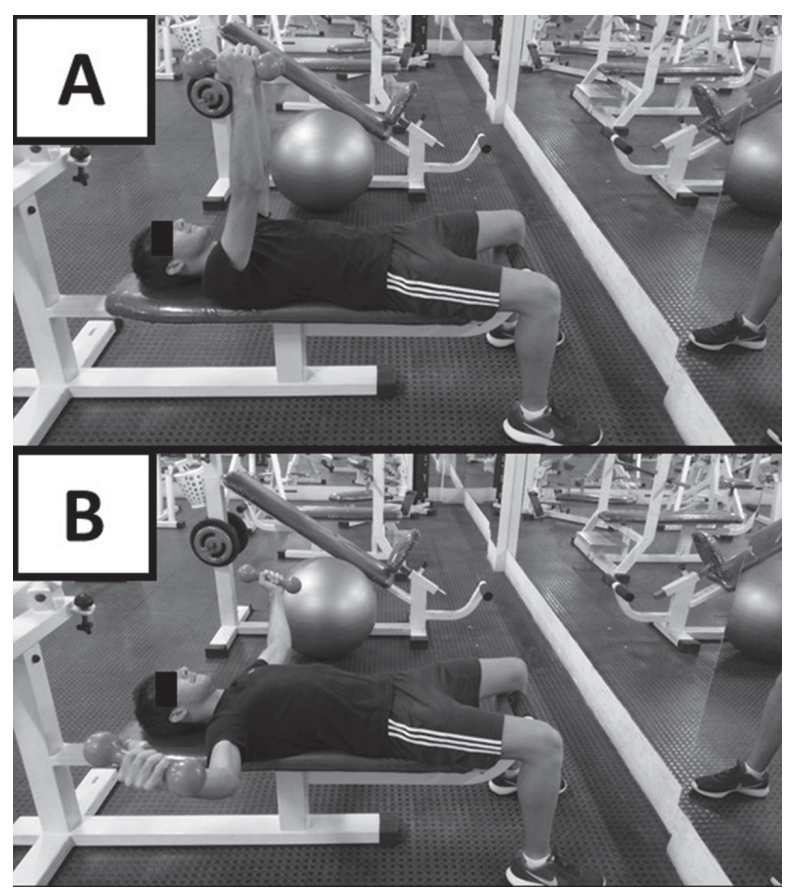

Figure 2. Side view of the movement's initial phase (A) and final phase (B) of the stable dumbbell fly exercise.

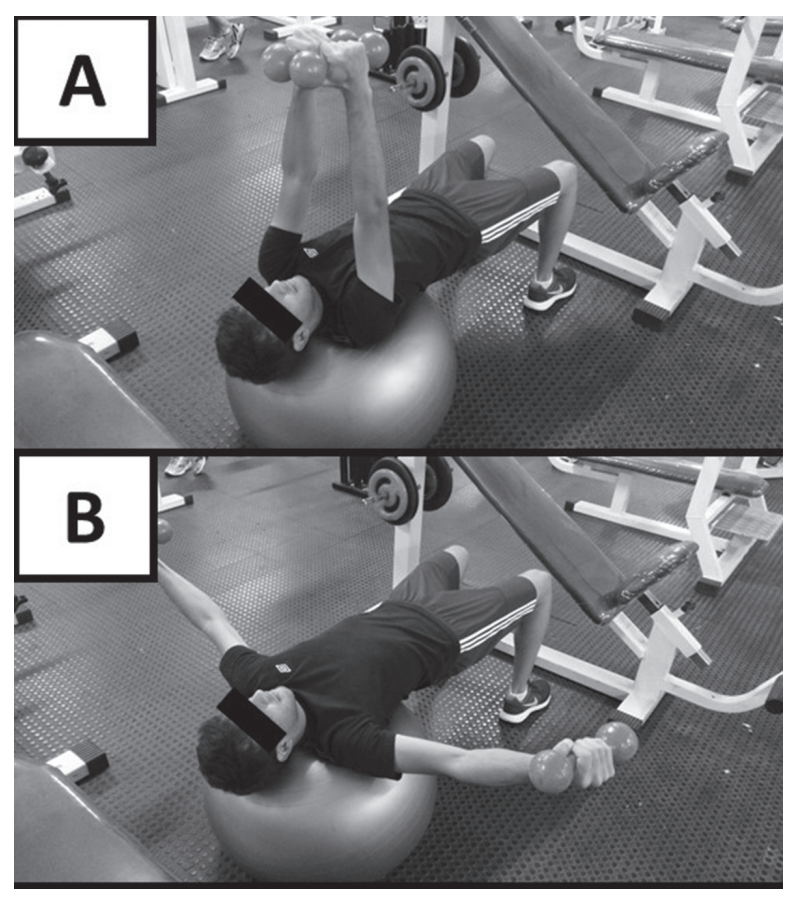

Figure 3. Side view of the movement's initial phase (A) and final phase (B) of the unstable dumbbell fly exercise.

\section{Data analyses}

Data were processed and analyzed using the software program Statistical Package for the Social Sciences (SPSS), version 16.0 (SPSS Inc., Chicago, IL, USA, Release 16.0.2, 2008). Normality was checked with the Shapiro-Wilk test. A two-way analysis of ANOVA for repeated measures with principal factors conditions (A, B and C) and muscles 
(PM, DA, TB, SA) was used to compare the EMG activity during the bench press. A one-way ANOVA for repeated measures was used to compare the EMG activity during the repetitions. When $\mathrm{F}$ was significant, a Bonferroni post-hoc test was applied to identify the differences ( $\mathrm{p} \leq .05$ ). To complement the analysis, the effect size (ES) was presented.

\section{Results}

The different protocols applied did not affect the EMG activity between muscles (Figure 4), whereas there was no interaction between conditions and muscles $(\mathrm{F}(6,104)=1.489, \mathrm{p}=.189 ; \mathrm{ES}=0.079)$.

Data presented in Table 1 demonstrate that the different execution methods affected EMG activity of the muscles during the bench press $(\mathrm{F}(2,104)$ $=26.733 ; p=.001, E S=0.44)$. It was observed that the pre-activation method, performed on both surfaces, resulted in higher EMG activity for PM $\left(\mathrm{p} \leq .01 ; \mathrm{ES}_{\text {(traditional } \mathrm{x} \text { stable) }}=0.79 ; \mathrm{ES}_{\text {(traditional } \mathrm{x} \text { unstable) }}\right.$ $=0.77) ; \mathrm{DA}\left(\mathrm{p} \leq .02 ; \mathrm{ES}_{\text {(traditional x stable) }}=0.19 ; \mathrm{ES}_{\text {(traditional }}\right.$ $x$ unstable) $=0.26) ; \mathrm{TB}\left(\mathrm{p} \leq .02 ; \mathrm{ES}_{\text {(traditional } \mathrm{x} \mathrm{stable)}}=0.62\right.$; $\left.\mathrm{ES}_{\text {(traditional x unstable) }}=0.66\right)$, and $\mathrm{SA}\left(\mathrm{p} \leq .03 ; \mathrm{ES}_{\text {(traditional }}\right.$ $x$ stable) $\left.=0.40 ; \mathrm{ES}_{\text {(traditional x unstable) }}=0.45\right)$ muscles. How- ever, no differences were observed in EMG activity between the stable and unstable conditions ( $\mathrm{p} \geq .228$; $\mathrm{EF}=0.065$ ).

Analysis of the EMG activity level for each repetition showed that PM (F $(7,273)=36.336$, $\mathrm{p}=.001 ; \quad \mathrm{ES}=0.582), \quad \mathrm{DA} \quad(\mathrm{F} \quad(7,273)=27.329$, $\mathrm{p}=.001 ; \mathrm{ES}=0.412), \mathrm{TB}(\mathrm{F}(7,273)=27.826, \mathrm{p}=.001$; $\mathrm{ES}=0.416)$, and SA $(\mathrm{F}(7,273)=4.050, \mathrm{p}=.027$; $\mathrm{ES}=0.101)$ manifested different EMG activity levels over the repetitions. For all three protocols, EMG activity during the first repetition was increased for $\mathrm{PM}(\mathrm{p} \leq .03), \mathrm{AD}(\mathrm{p} \leq .04)$ and TB $(\mathrm{p} \leq .03)$, whereas stabilization occurred at the fifth repetition for PM $(\mathrm{p} \geq .28)$ and DA $(\mathrm{p} \geq .19)$ and at the sixth repetition for TB ( $\mathrm{p} \geq .12)$. SA manifested unusual EMG activity levels across all the repetitions and did not present a pattern for EMG signals.

There was no interaction among the different protocols and the EMG activity along the repetitions for any assessed muscle as follows: PM (F $(14.273)=0.858, \mathrm{p}=.605 ; \mathrm{ES}=0.042), \mathrm{DA}(\mathrm{F}(14.273)$ $=0.655 ; \mathrm{p}=.817, \mathrm{ES}=0.033), \mathrm{TB}(\mathrm{F}(14.273)=0.346$, $\mathrm{p}=.987, \mathrm{ES}=0.017)$ and $\mathrm{SA}(\mathrm{F}(14.273)=1.181, \mathrm{p}=.289$, $\mathrm{ES}=0.057$ ) (Figure 5).

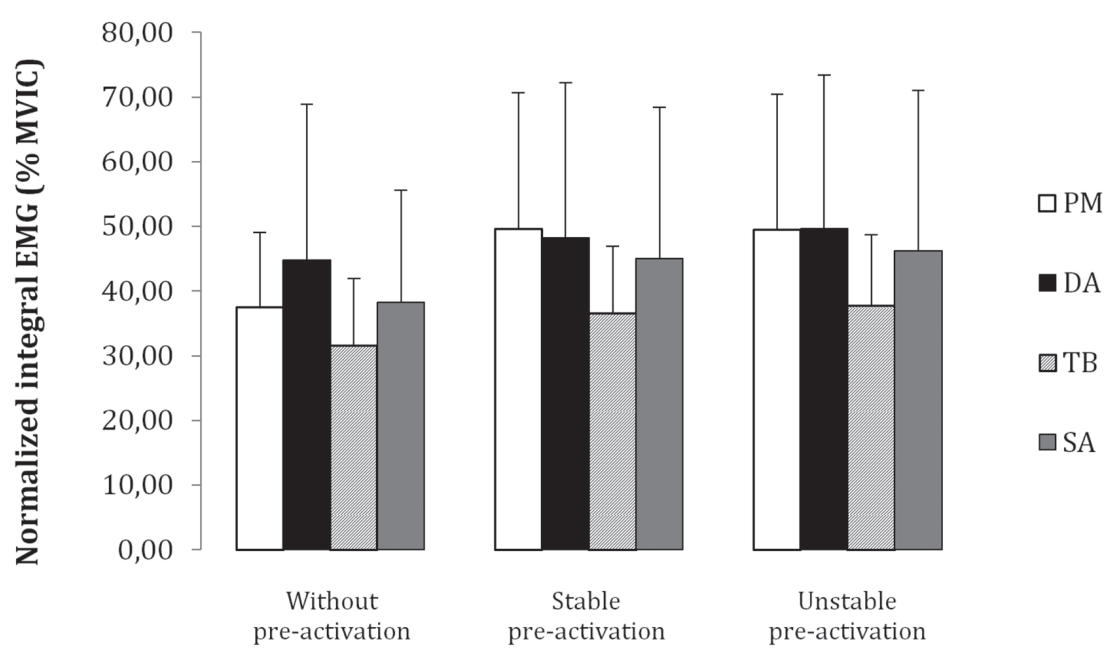

Figure 4. Relationship of EMG activity among the evaluated muscles in different methods. MVIC = maximal voluntary isometric contraction; $P M=m$. pectoralis maior; $D A=m$. deltoid anterior; $T B=m$. triceps brachii; $S A=m$. serratus anterior. $p \leq .05$

Table 1. Mean values and standard deviations of normalized EMG integral (\% maximal voluntary isometric contraction) corresponding to EMG activity of the muscles evaluated during the realization of the traditional and pre-activation methods (stable and unstable)

\begin{tabular}{lccc}
\hline Muscles & Traditional & Pre-activation stable & Pre-activation unstable \\
\hline Pectoralis maior & $37.53 \pm 11.52$ & $49.62 \pm 21.01^{*}$ & $49.43 \pm 21.01^{*}$ \\
Deltoid anterior & $44.76 \pm 24.16$ & $48.26 \pm 23.89^{*}$ & $49.61 \pm 23.77^{*}$ \\
Triceps brachii & $31.51 \pm 10.47$ & $36.52 \pm 10.39^{*}$ & $37.72 \pm 10.98^{*}$ \\
Serratus anterior & $38.23 \pm 17.36$ & $44.98 \pm 23.38^{*}$ & $46.18 \pm 24.79^{*}$ \\
\hline
\end{tabular}

* Statistical difference compared to the traditional method $(p \leq .05)$. 

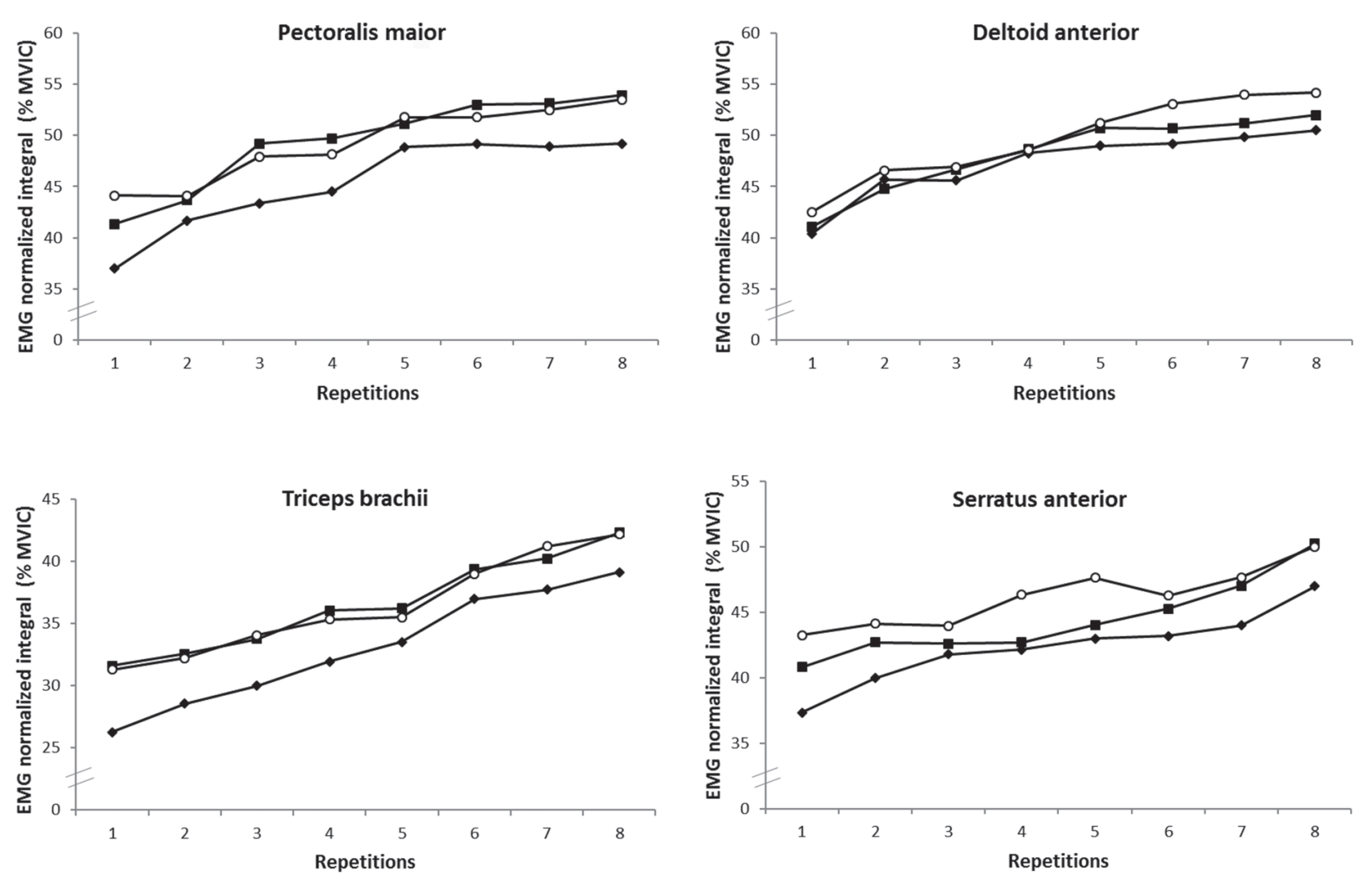

$\rightarrow$ Without pre-activation

Stable pre-activation

$\multimap-$ Unstable pre-activation

Figure 5. EMG activity trend during the intermediate repetitions, for the evaluated muscles in different methods.

\section{Discussion and conclusions}

The present study compared the EMG activity of the upper limb muscles and shoulder girdle during the pre-activation performance method of resistance training executed on a stable and unstable surface. The initial hypothesis of the study was that the pre-activation, promoted by a single-joint exercise, along with the unstable surface would increase the EMG activity of the primary agonists in the subsequent multi-joint exercise. In fact, for both surfaces, when compared to the individual multijoint exercise, there was an increase in the amplitude of the EMG signal for PM and DA under the conditions where the dumbbell fly exercise was previously performed.

The data from this study differ from those observed in some other studies (Augustsson, et al., 2003; Brennecke, et al., 2009; Fisher, Carlson, Steele, \& Smith, 2014; Gentil, et al., 2007). A technique frequently used by weight trainers involves a single-joint exercise followed immediately by a related multijoint exercise (e.g., a knee extension exercise followed by a leg press exercise in which the pre-exhaustion method had no effect). This divergence can be explained by the high intensity load applied to the single-joint exercise in these studies. According to Júnior et al. (2010) when low/ moderate loads are used in the first exercise, the preactivation of the muscles occurs, and this improves performance in the following exercise.

However, although the results are apparently in favor of pre-activation, the analysis of EMG activity along the repetitions and the evaluation of the assisting and stabilizing muscle EMG activity, not observed in previous studies, question the validity of this method. According to the assumption of the pre-activation method, it was expected that, for the conditions in which there was a prior activation of the primary muscles promoted by the dumbbell fly exercise, there would be an increase followed by the stabilization of PM and DA EMG activity from the first repetition of the bench press exercise. According to Enoka (2000), force reaches a plateau when there are no more motor units to be recruited, and there occurs stabilization in the frequency of the electrical impulses, captured by the EMG signals. Analyzing the EMG activity along the repetitions of the bench press exercise, it was found that the prime mover muscles were only stabilized in the fifth repetition and that this pattern was common to all conditions, regardless of the prior execution of the dumbbell fly exercise. 
While it is possible to speculate that this late stabilization was caused by the low intensity employed in the single-joint exercise, it is important to consider that the adopted protocol was similar to that executed by Júnior et al. (2010), who reported the increased EMG activity with the use of moderate intensity. Although the study has not explored the stabilization of EMG activity throughout the repetitions, it is the first to demonstrate positive effects of the pre-exhaustion method, contrary to what has been observed in previous studies with maximum intensities, independent of body segment (Augustsson, et al., 2003; Brennecke, et al., 2009; Fisher, et al., 2014; Gentil, et al., 2007).

Another fact that may question the efficiency of the pre-activation method is the increased EMG activity of the auxiliary (TB) and stabilizer (SA) muscles observed in our study. For validity of the method to be accepted, only the EMG activity of the muscles activated during the dumbbell fly (PM and DA) should have increased. The comparison of these results with other studies is limited because the only study that followed similar procedures in the pre-activation method (Júnior, et al., 2010) only assessed the EMG activity of the primary agonist muscle. Accordingly, it can be speculated that similar behavior would be observed for secondary agonist muscles if these were evaluated, or that the execution of any non-specific exercise would promote the same behavior, regardless of the monojoint and multi-joint exercise combination for the same muscle group. Therefore, it is suggested that a new research should explore the execution of monoand multi-joint exercises for the same muscle group in different combinations.

The second hypothesis of this study was that the use of an unstable surface for the pre-exhaustion or pre-activation method, not evaluated in previous studies, could promote a greater EMG activity in the multi-joint exercise. However, the results showed that the use of an unstable surface in the dumbbell fly exercise did not change the muscle activation levels in the bench press exercise.

It is important to consider that the study by Melo et al. (2014), which compared EMG activity in the dumbbell fly exercise using stable and unstable surfaces with the same sample as this study, found that instability increased neuromuscular demand, generating a greater activation of agonist (PM and DA) and stabilizer (SA) muscles. Similarly, other studies also found that strength exercises, when performed on unstable surfaces, promote a greater EMG activity, even with lower intensity (Behm, Anderson, \& Curnew, 2002; Campbell, Kutz, Morgan, Fullenkamp, \& Ballenger, 2014; Marshall \& Murphy, 2006; McBride, Cormie, \&
Deane, 2006; Nejc, Loefler, Cvecka, Sedliak, \& Kern, 2013).

Performing exercises on unstable surfaces is characterized as a challenging task for the neuromotor system in that it stresses the proprioceptive system at a greater magnitude, generating greater EMG demands during movement execution. However, based on the results of the study by Melo et al. (2014) and the present study, it can be said that there is an increase in EMG activity when a strength exercise with a rotational load direction (not linear) (Lephart \& Henry, 1996) is performed on an unstable surface, but this benefit is not transferred to a subsequent exercise executed on a stable surface. In this sense, further studies should explore whether different responses would be observed if the multi-joint exercises were also performed under an unstable condition.

Our results indicate that executing a previous exercise resulted in the increased EMG activity of all the muscles evaluated (prime movers, a secondary mover and a stabilizer) during the subsequent exercise execution. However, as there was no exclusive increase for the primary agonist muscles in the multi-joint exercise, this increase cannot be attributed to the muscle activation promoted by a specific exercise, but rather to the completion of two consecutive exercises without a resting interval. Furthermore, it was observed that the use of an unstable surface was not sufficient to generate higher levels of EMG activity or to modify the recruitment of the muscles tested. Thus, it can be suggested that the differences observed in the EMG activity levels in different protocols should be attributed to the execution of the previous exercise, not to the use of instability.

Importantly, the analysis of the EMG activity of different muscles during each repetition, presented in this study, allows advances in the discussion about the effectiveness of the pre-activation method. In this sense, considering this information allows a more careful observation since the average EMG signal at the end of the series, reported in previous studies by Gentil et al. (2007), is not sufficient to guarantee the effectiveness of the method. Finally, it should be noted that gaps identified in our study should be explored in further research. Among them are the use of other types of exercise as a previous activation strategy, or the use of unstable surfaces in a multi-joint exercise with the pre-activation method.

In practical terms, the pre-exhaustion method or pre-activation is commonly used in the prescription of RT in order to increase the activation of agonist muscles. However, according to the results of this study, it is recommended to use pre-activation to 
raise the EMG activity of all muscles involved in subsequent exercise. In addition, we emphasize that this recommendation is valid for single-joint exercises performed at $30 \%$ of maximum load, regardless of the type of surface on which they are executed.

\section{References}

ACSM. (2009). American College of Sports Medicine. Position stand: Progression models in resistance training for healthy adults. Medicine and Science in Sports and Exercise, 41(3), 687-708.

Andrade, R., Araújo, R., Tucci, H., Martins, J., \& Oliveira, A. (2011). Coactivation of the shoulder and arm muscles during closed kinetic chain exercises on an unstable surface. Singapore Medical Journal, 52(1), 35-41.

Augustsson, J., Thomee, R., Hornstedt, P., Lindblom, J., Karlsson, J., \& Grimby, G. (2003). Effect of pre-exhaustion exercise on lower-extremity muscle activation during a leg press exercise. Journal of Strength and Conditioning Research, 17(2), 411-416.

Behm, D.G., Anderson, K., \& Curnew, R.S. (2002). Muscle force and activation under stable and unstable conditions. Journal of Strength and Conditioning Research, 16(3), 416-422.

Behm, D., \& Colado, J.C. (2012). The effectiveness of resistance training using unstable surfaces and devices for rehabilitation. International Journal of Sports Physical Therapy, 7(2), 226-241.

Brennecke, A., Guimarães, T.M., Leone, R., Cadarci, M., Mochizuki, L., Simão, R., et al. (2009). Neuromuscular activity during bench press exercise performed with and without the preexhaustion method. Journal of Strength and Conditioning Research, 23(7), 1933-1940.

Brown, L.E., \& Weir, J.P. (2001). ASEP procedures recommendation I: Accurate assessment of muscular strength and power. Professionalization of Exercise Physiology. Journal of Exercise Physiology Online, 4(3), 1-21

Campbell, B.M., Kutz, M.R., Morgan, A.L., Fullenkamp, A.M., \& Ballenger, R. (2014). An evaluation of upperbody muscle activation during coupled and uncoupled instability resistance training. Journal of Strength and Conditioning Research, 28(7), 1833-1838.

de Araújo, R.C., Tucci, H.T., de Andrade, R., Martins, J., Bevilaqua-Grossi, D., \& de Oliveira, A.S. (2009). Reliability of electromyographic amplitude values of the upper limb muscles during closed kinetic chain exercises with stable and unstable surfaces. Journal of Electromyography and Kinesiology, 19(4), 685-694.

Enoka, R.M. (2000). Bases neuromecânicas da cinesilogia. São Paulo: Manole.

Fisher, J.P., Carlson, L., Steele, J., \& Smith, D. (2014). The effects of pre-exhaustion, exercise order, and rest intervals in a full-body resistance training intervention. Applied Physiology, Nutrition, and Metabolism, 39(11), 1265-1270.

Fleck, S., \& Kraemer, W. (2004). Designing resistance training programs ( $3^{\text {rd }}$ ed.). Champaign, IL: Human Kinetics.

Gentil, P., Oliveira, E., Júnior, V.D.A.R., do Carmo, J., \& Bottaro, M. (2007). Effects of exercise order on upper-body muscle activation and exercise performance. Journal of Strength and Conditioning Research, 21(4), 1082-1086.

Hermens, H.J., Freriks, B., Disselhorst-Klug, C., \& Rau, G. (2000). Development of recommendations for SEMG sensors and sensor placement procedures. Journal of Electromyography and Kinesiology, 10(5), 361-374.

Júnior, R., Valdinar, A., Bottaro, M., Pereira, M.C., Andrade, M.M., Júnior, P., et al. (2010). Electromyographic analyses of muscle pre-activation induced by single joint exercise. Brazilian Journal of Physical Therapy, 14(2), 158-165.

Kendall, H.O., Kendall, F.P., \& Wadsworth, G.E. (1973). Muscles, testing and function. American Journal of Physical Medicine and Rehabilitation, 52(1), 43.

Lephart, S.M., \& Henry, T.J. (1996). The physiological basis for open and closed kinetic chain rehabilitation for the upper extremity. Journal of Sport Rehabilitation, 5, 71-87.

Marshall, P.W., \& Murphy, B.A. (2006). Increased deltoid and abdominal muscle activity during Swiss ball bench press. Journal of Strength and Conditioning Research, 20(4), 745-750.

McBride, J.M., Cormie, P., \& Deane, R. (2006). Isometric squat force output and muscle activity in stable and unstable conditions. Journal of Strength and Conditioning Research, 20(4), 915-918.

Melo, B., Pirauá, A., Beltrão, N., Pitangui, A.C., \& Araújo, R. (2014). A utilização de superfície instável aumenta a atividade eletromiográfica dos músculos da cintura escapular no exercício crucifixo. [The use of unstable surface increases the electromyographic activity of the shoulder girdle muscles in dumbbell fly exercise. In Portuguese.]. Revista Brasileira de Atividade Física \& Saúde, 19(3), 342.

Šarabon, N., Loefler, S., Cvecka, J., Sedliak, M., \& Kern, H. (2013). Strength training in elderly people improves static balance: A randomized controlled trial. European Journal of Translational Myology, 23(3), 85-89.

Pirauá, A.L.T., Pitangui, A.C.R., Silva, J.P., dos Passos, M.H.P., de Oliveira, V.M.A., Batista, L.d.S.P., et al. (2014). Electromyographic analysis of the serratus anterior and trapezius muscles during push-ups on stable and unstable bases in subjects with scapular dyskinesis. Journal of Electromyography and Kinesiology, 24(5), 675-681. 
Ribeiro, A.S., Silva, D.R.P. d., Nascimento, M.A. d., Avelar, A., Ritti-Dias, R.M., \& Cyrino, E.S. (2013). Efeito da manipulação da ordem dos exercícios de força utilizando o sistema tri-set. [Effect of the manipulation of exercise order in the tri-set training system. In Portuguese.] Revista Brasileira de Cineantropometria \& Desempenho Humano, 15(5), 527-534.

Tucci, H.T., Ciol, M.A., De Araújo, R.C., De Andrade, R., Martins, J., McQuade, K.J., et al. (2011). Muscle activation of selected shoulder muscles during unilateral wall and bench press tasks under submaximal isometric effort. Journal of Orthopaedic and Sports Physical Therapy, 41(7), 520-525.

Submitted: July 9, 2016

Accepted: March 6, 2017

Published Online First: October 12, 2017

Correspondence to:

Rodrigo Cappato de Araújo

Universidade de Pernambuco, Campus Petrolina

BR $203 \mathrm{Km} 2 \mathrm{~S} / \mathrm{N}$, Vila Eduardo

Cep: 56300-000 - Petrolina, PE - Brazil

Phone/Fax: (55 87) 3866-6468

E-mail: rodrigo.cappato@upe.br

\section{Acknowledgments}

Funding for this project came from Coordenação de Aperfeiçoamento de Pessoal de Nivel Superior, Brazil. Data gathering of this research relied on the collaboration of Bruno Melo. 